Combining Eqs. [3] and [4] yields a definition of $\mathrm{K}_{\mathrm{a}}$ independent of the pulse velocity $\mathrm{V}_{\mathrm{p}}$ (Stein and Kane, 1983; Van Loon et al., 1990):

\section{Time-domain Reflectometry for Measuring Water Content of Organic Growing Media in Containers}

\author{
Tomasz Anisko ${ }^{1}$, D. Scott NeSmith ${ }^{2}$, and Orville M. Lindstrom ${ }^{3}$ \\ Department of Horticulture, University of Georgia, Georgia Experiment \\ Station, Griffin, GA 30223
}

Additional index words. dielectric constant, calibration equation

\begin{abstract}
The time-domain reflectometry (TDR) method of measuring water content has been applied to mineral soils but not to organic growing media. We investigated the applicability of TDR for measuring the water content of organic media in containers. TDR calibration was conducted for sand, peat, composted pine bark, sand and peat mix, sand and bark mix, and a commercial growing medium (Metro Mix 300). Regression analysis of volumetric water content was conducted with the ratio of apparent : physical length of the probe $\left(L_{\mathrm{a}}: L\right)$ as an independent variable. The calibration curve for Metro Mix 300 was compared to curves generated for a range of soils by other investigators. Additionally, water-content and $L_{a}$ : $L$ changes were monitored in Metro Mix 300 for 10 months and were compared to predicted values from the calibration curve. Organic media had a higher water content than sand for the same $L_{a}: L$ value. Equations developed by previous authors generally underestimated water content when compared with the calibration curve for Metro Mix 300. We attribute this difference to a large fraction of highly decomposed organic matter or vermiculite and, thus, to the presence of more bound water. Specific calibration of TDR may be required to determine the absolute water content of organic growing media.
\end{abstract}

Time-domain reflectometry (TDR) is a relatively new method for measuring soil water content. It possesses distinct advantages, such as safety of operation, portability, easy installation, and rapid data collection, that have led to its increased usage. TDR is an accurate and nondestructive method that allows continuous measurements over the full soil moisture range. Most of the reports that describe TDR are field studies, and only recently have others (Richardson et al., 1992) explored its use in containers.

TDR measures the apparent dielectric permittivity of soil to the electromagnetic signals at microwave (MHz-GHz) frequencies (Baker, 1990; Van Loon, 1990). The method uses a cable tester that consists of a pulse generator, a sampler that produces low-frequency signals, and an oscilloscope that displays the sampler output (Baker, 1990). Electromagnetic pulses travel down a transmission line that terminates in a parallel pair of stainless steel waveguides inserted into soil (Baker, 1990). The oscilloscope screen displays the reflected pulses that exhibit perturbations in the transmission line where impedance changes

Received for publication 29 Nov. 1993. Accepted for publication 16 Apr. 1994. Use of trade names does not imply endorsement of the products named nor criticism of similar ones not named. The cost of publishing this paper was defrayed in part by the payment of page charges. Under postal regulations, this paper therefore must be hereby marked advertisement solely to indicate this fact.

${ }^{1}$ Graduate Research Assistant.

${ }^{2}$ Assistant Professor.

${ }^{3}$ Associate Professor.

occur. These are 1) the point of pulse reflection at the juncture of the cable with the steel waveguides inserted into a soil and 2) the point of pulse reflection at the end of the waveguides (Baker, 1990). These two points, together with the known length of the waveguides, allow calculation of the pulse velocity, $\mathrm{V}_{\mathrm{p}}$, relative to the velocity of electromagnetic radiation in free space $\left(\mathrm{c}=3 \times 10^{8} \mathrm{~m} \cdot \mathrm{s}^{-1}\right)$ (Baker, 1990). The apparent dielectric constant, $\mathrm{K}_{\mathrm{a}}$, can be approximated by the equation (Baker, 1990):

The velocity of pulse $\left(\mathrm{V}_{\mathrm{p}}\right)$ along a transmission line is given by the equation (Stein and Kane, 1983):

where L is the physical length of the waveguides and $t$ is the time of propagation, which depends on dielectric properties of the medium surrounding the waveguides. Combining Eqs. [1] and [2] gives (Stein and Kane, 1983):

To calculate $\mathrm{K}_{\mathrm{a}}$, the velocity of pulse, $\mathrm{V}_{\mathrm{p}}$, is set on the instrument to $\mathrm{c}$, and the apparent length of the waveguides, $L_{a}$, is read on the oscilloscope. Therefore, the following conditions prevail (Stein and Kane, 1983)

HortScience, Vol. 29(12), December 1994 where $\mathrm{L}$ is the true length of the TDR waveguides in soil and $L_{a}$ is the apparent length as measured with the TDR device.

The dielectric constants for oven-dried soil, air, and water are $\approx 4,1$, and 80 , respectively, so that $\mathrm{K}_{\mathrm{a}}$ for soil strongly depends on water content (Baker, 1990). The relationship between the dielectric constant and water content $(\theta)$ is not purely linear, although it is nearly so over much of the range of water contents encountered in the field (Baker, 1990). Several authors (Pepin et al., 1992; Roth et al., 1992; Stein and Kane, 1983; Topp et al., 1980) established empirical relationships between $\theta$ and $\mathrm{K}_{\mathrm{a}}$ for various types of soils in the form of polynomial equations (Table 1). Others report linear calibration equations stated in terms of the true waveguide length, $\mathrm{L}$, and the apparent waveguide length, $\mathrm{L}_{\mathrm{a}}$ (Ledieu et al., 1986), or the pulse velocity, $\mathrm{V}_{\mathrm{p}}$ (Herkelrath et al., 1991). Topp et al. (1980) suggested that specific calibration of TDR is unnecessary for most soils. However, organic soils are a notable exception to general application of TDR measurements and require a separate calibration equation, probably due to more bound water (Herkelrath et al., 1991; Roth et al., 1992; Topp et al., 1980).

Containerized horticultural production is based on artificial soilless growing mixes composed primarily of various organic materials. A nondestructive and accurate method of measuring moisture content is strongly desired by researchers and producers. This study evaluates the applicability of TDR and alreadydeveloped calibration equations for measuring the water content of organic growing media in containers.

\section{Materials and Methods}

The TDR probes were stainless steel welding rods ( $3.2 \mathrm{~mm}$ in diameter) cut as pairs to 15 $\mathrm{cm}$ in length (L). A single pair of rods was inserted vertically into each medium at a parallel distance of $50 \mathrm{~mm}$. This procedure resulted in a single water-content reading integrated over the depth traversed by the probes. The apparent length of the probes $\left(\mathrm{L}_{\mathrm{a}}\right)$ in the medium was determined with a Tektronix 1502C Metallic Time Domain Reflectometer (Tektronix, Beaverton, Ore.).

TDR calibration was conducted for sand, peat, composted pine bark, 1 sand : 1 peat mix (v/v), 1 sand : 1 bark mix (v/v), and a commercial growing medium (Metro Mix 300; Grace Sierra Horticultural Co., Milpitas, Calif.). Metro Mix 300 is composed of sphagnum peat, composted pine bark, processed bark ash, vermiculite, perlite, and sand. Each medium was placed in twelve 2.2-liter pots and then pressed by hand to imitate settling. The following day, pots were watered in increments of $\approx 5 \%$ volumetric to give pots with a 
water content ranging from near air-dry to saturation. One day later, a TDR reading was taken in each pot and each pot was weighed for gravimetric determination of water content. Calibration curves for each medium were generated by regressing volumetric water content against the $\mathrm{L}_{\mathrm{a}}$ : $\mathrm{L}$ ratio. The calibration curve for Metro Mix 300 was compared to curves generated from the equations of Topp et al. (1980), Stein and Kane (1983), Ledieu et al. (1986), Herkelrath et al. (1991), Pepin et al. (1992), and Roth et al. (1992). To make necessary comparisons, the equation of Herkelrath et al. (1991) was altered in terms of $\mathrm{L}_{\mathrm{a}}: \mathrm{L}$ ratio rather than $\mathrm{V}_{\mathrm{p}}$.

Two-year-old 'Yaku Princess' Rhododendron plants were grown in 2.2-liter pots containing Metro Mix 300 growing medium. The 15-cm TDR probes were inserted as described above. TDR measurements and pot weighings were conducted twice a week between Aug. 1992 and June 1993. During this time, no visible growth of the plants was observed, except for late May and June, when new stem development began. We assumed that the pot weight changes between measurements ( 3 to 4 days) were primarily a result of changes in water content rather than plant biomass changes. Regression analysis of volumetric water-content changes between measurements vs. $\mathrm{L}_{\mathrm{a}}$ : $\mathrm{L}$ ratio changes was performed and compared with earlier calibration data.

\section{Results and Discussion}

Comparison of calibration curves revealed differences among media, with organic media (peat, bark, Metro Mix 300) having a higher water content than sand for the same $\mathrm{L}_{\mathrm{a}}$ : $\mathrm{L}$ value (Table 2, Fig. 1). Mixes of sand with peat and bark had properties intermediate when compared to the individual basic components. Metro Mix 300 had the highest water content of all media at any given $\mathrm{L}_{\mathrm{a}}: \mathrm{L}$ value. Differences between organic media and sand can be attributed to higher organic matter content in the former and, thus, to a larger fraction of bound water with a lower $\mathrm{K}_{\mathrm{a}}$. Several authors (Dasberg and Hopmans, 1992; Dobson et al., 1985; Wang and Schmugge, 1980) showed that $K_{a}$ values of fine-textured soils were lower than those of coarse-textured soils at equal water content, the difference being explained by the presence of bound water with a low $\mathrm{K}_{\mathrm{a}}$. Soil particles that have larger surface areas hold more water in the bound state than others. Bound water molecules close to the surface of soil particles are less mobile than free water and have dielectric properties similar to ice (i.e., $\mathrm{K}_{\mathrm{a}}=3$ ). Similarly, Topp et al. (1980) attributed anomalous dielectric properties of organic soils to the appreciably greater amount of bound water present. However, the distinct readings for Metro Mix 300 cannot be explained by the bulk amount of organic matter alone, which is similar to that for peat and bark. Vermiculite, one of the components of Metro Mix 300, was reported by Topp et al. (1980) to have dielectric properties similar to an organic soil. Apparently, a large fraction of highly decomposed organic matter or ver-

Table 1. Coefficients of the calibration equations representing the empirical relationship between volumetric water content $(\theta)$ and apparent dielectric constant $\left(\mathrm{K}_{\mathrm{a}}\right)$ for various media.

\begin{tabular}{lccccl}
\hline & \multicolumn{4}{c}{ Coefficients of calibration equation } \\
Medium & $\alpha=\alpha \times 10^{-2}+\beta \times 10^{-2} \times \mathrm{K}_{\mathrm{a}}+\gamma \times 10^{-4} \times \mathrm{K}_{\mathrm{a}}{ }^{2}+\delta \times 10^{-6} \times \mathrm{K}_{\mathrm{a}}{ }^{3}$ & \\
\cline { 2 - 5 } Mineral soil & $\alpha$ & $\beta$ & $\gamma$ & $\delta$ & Reference \\
Organic soil & -5.3 & 2.92 & -5.5 & 4.3 & Topp et al., 1980 \\
& -2.52 & 4.15 & -14.4 & 22.0 & Stein and Kane, 1983 \\
Peat & -2.33 & 2.85 & -4.31 & 3.04 & Roth et al., 1992 \\
& 8.5 & 1.92 & -0.95 & 0 & Pepin et al., 1992 \\
\hline
\end{tabular}

Table 2. Regression coefficients for calibration curves of six media and coefficients of Ledieu et al. (1986) and Herkelrath et al.(1991).

\begin{tabular}{lcrr}
\hline \hline & \multicolumn{3}{c}{ Regression coefficients of calibration equation } \\
\cline { 2 - 4 } Medium & \multicolumn{1}{c}{$\begin{array}{c}\theta=\alpha+\beta\left(\mathrm{L}_{\mathrm{a}}: \mathrm{L}\right) \\
\text { Metro Mix 300 }\end{array}$} & \multicolumn{1}{c}{$\beta$} & $r^{2}$ \\
Peat & $0.0383 \pm 0.0214$ & $0.1251 \pm 0.0057$ & 0.94 \\
Bark & $-0.1543 \pm 0.0235$ & $0.1427 \pm 0.0152$ & 0.90 \\
Sand & $-0.0800 \pm 0.0171$ & $0.1103 \pm 0.0182$ & 0.79 \\
Peat-sand & $-0.3255 \pm 0.0126$ & $0.1635 \pm 0.0074$ & 0.98 \\
Bark-sand & $-0.2376 \pm 0.0090$ & $0.1502 \pm 0.0060$ & 0.98 \\
Mineral soil (Ledieu et al., 1986) & $-0.1198 \pm 0.0124$ & $0.1251 \pm 0.0177$ & 0.83 \\
Forest soil (Herkelrath et al., 1991) & -0.1760 & 0.1140 & --- \\
\hline
\end{tabular}

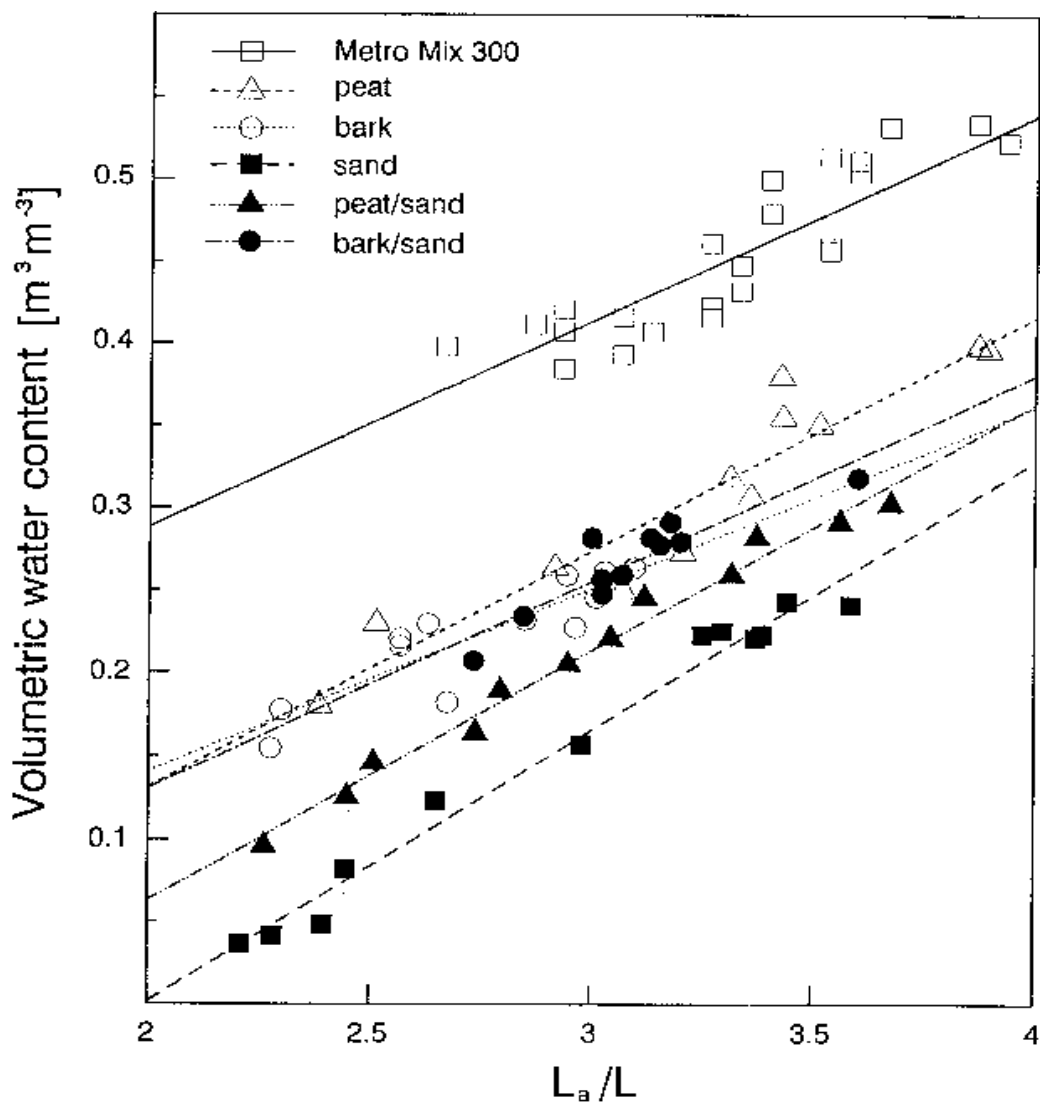

Fig. 1. Time-domain reflectometry calibration curves for measuring water content in Metro Mix 300, peat, bark, sand, peat-sand mix, and bark-sand mix.

miculite, or both, and thus the presence of more bound water, may influence the TDR relationship, but this relationship remains to be investigated.

Equations developed by Topp et al. (1980), Ledieu et al. (1986), and Herkelrath et al. (1991) for mineral soils; by Stein and Kane (1983) and Roth et al. (1992) for organic soils; and by Pepin et al. (1992) for peat generally underestimated water content when compared with the calibration curve for Metro Mix 300 (Fig. 2). Similar slopes of these curves suggest that water-content changes could be measured with any of the equations. However, calibration is necessary to determine the intercept for absolute water-content values. Monitoring water content in pots with 'Yaku Princess' Rhododendron for 10 months demonstrated a relationship between water-content changes and $L_{a}: L$ ratio $(\beta=0.1215 \pm 0.0027)$ (Fig. 3) 


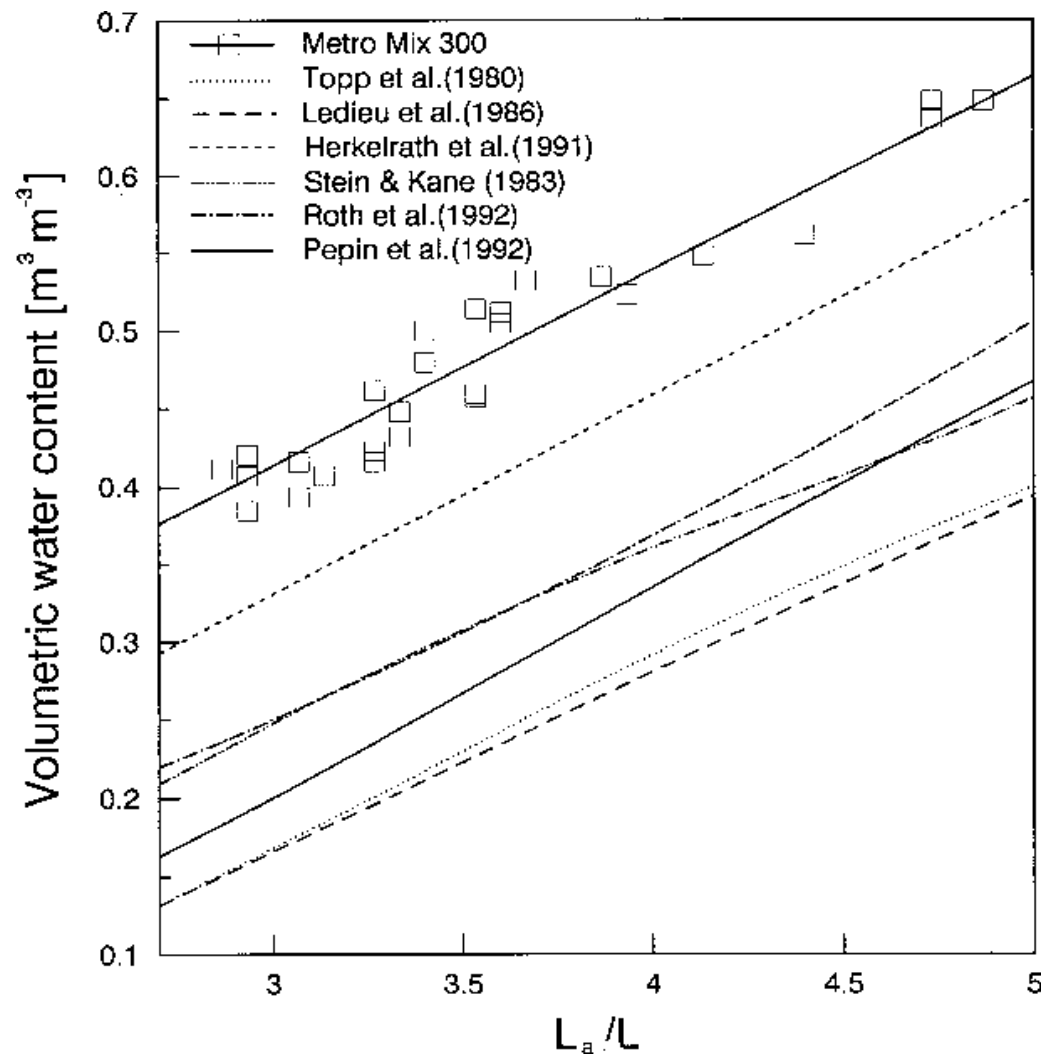

Fig. 2. TDR calibration curve for Metro Mix 300 compared to calibration curves of Topp et al. (1980), Ledieu et al. (1986), Herkelrath et al. (1991), Stein and Kane (1983), Roth et al. (1992), and Pepin et al. (1992).

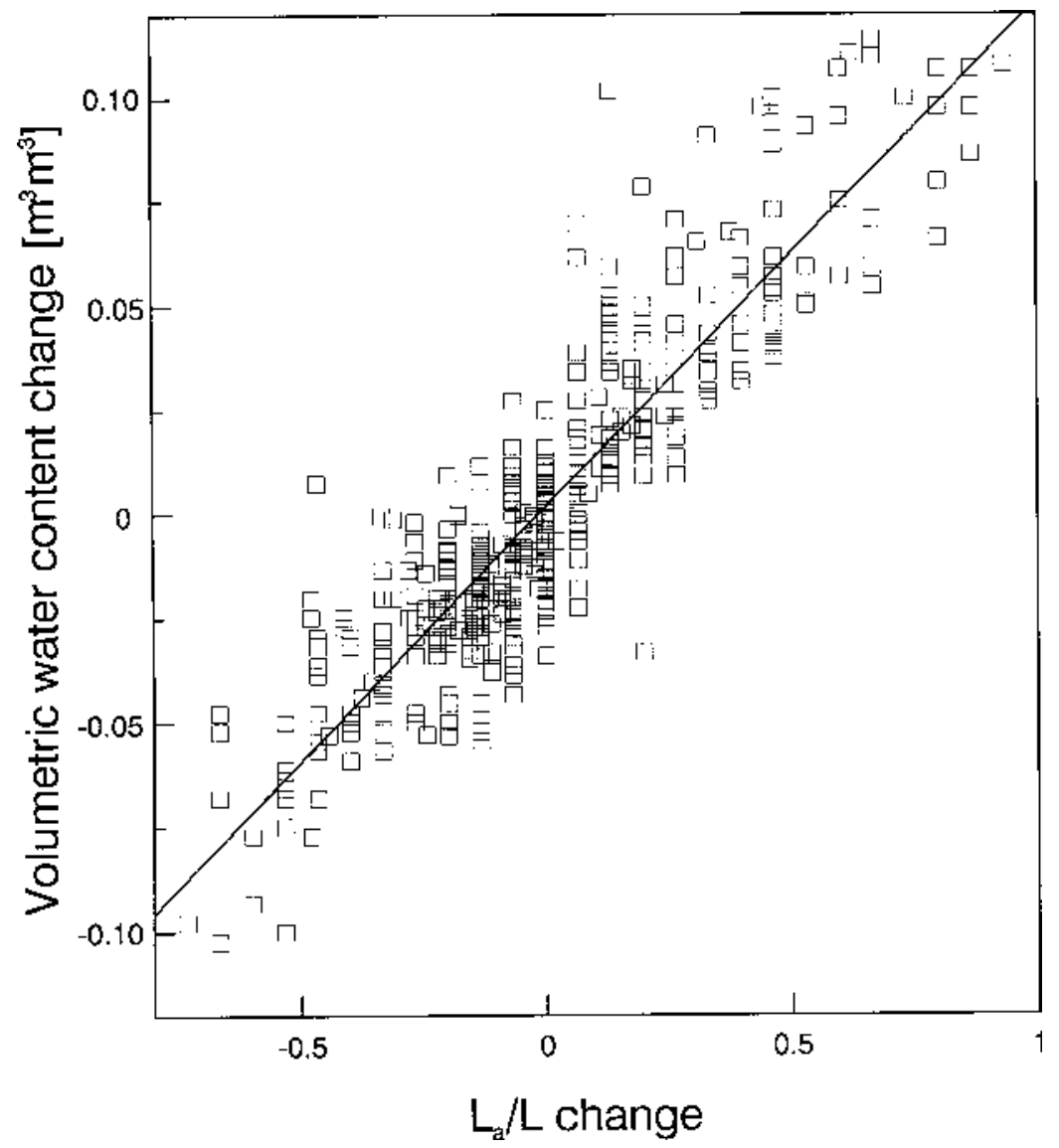

Fig. 3. Regression line of water content change vs. $L_{a}$ : L ratio change for Metro Mix $300(y=0.0015+$ $0.1215 x)$. virtually identical to that of the calibration curve $(\beta=0.1251 \pm 0.0057)$ (Table 2$)$.

This research demonstrates that TDR can be used to monitor the water content of organic media for containerized plants. Some specific calibration may be required if absolute water content needs to be determined. This procedure can be simplified by using the $\mathrm{L}_{\mathrm{a}}$ : L ratio in the form of a linear equation. However, the current equations will likely be adequate for monitoring water-content changes in organic media. This method may be particularly useful for researchers concerned with watering regimes of container-produced plants.

\section{Literature Cited}

Baker, J.M. 1990. Measurement of soil water content. Remote Sensing Rev. 5(1):263-279.

Dasberg, S. and J.W. Hopmans. 1992. Time domain reflectometry calibration for uniformly and nonuniformly wetted sandy and clayey loam soils. Soil Sci. Soc. Amer. J. 56:1341-1345.

Dobson, M.C., F.T. Ulaby, M.T. Hallikainen, and M.A. El-Rayes. 1985. Microwave dielectric behavior of wet soil-Part II: Dielectric mixing models. IEEE Trans. Geoscience Remote Sensing GE-23:35-46.

Herkelrath, W.N., S.P. Hamburg, and F. Murphy. 1991. Automatic, real-time monitoring of soil moisture in a remote field area with time domain reflectometry. Water Resources Res. 27:857864.

Ledieu, J., P. de Ridder, P. de Clerck, and S. Dautrebande. 1986. A method of measuring soil moisture by time-domain reflectometry. $\mathrm{J}$. Hydrol. 88:319-328.

Pepin, S., A.P. Plamondon, and J. Stein. 1992. Peat water content measurement using time domain reflectometry. Can. J. For. Res. 22:534-540.

Richardson, M.D., C.A. Meisner, C.S. Hoveland, and K.J. Karnok. 1992. Time domain reflectometry in closed container studies. Agron. J. 84:1061-1063.

Roth, C.H., M.A. Malicki, and R. Plagge. 1992. Empirical evaluation of the relationship between soil dielectric constant and volumetric water content as the basis for calibrating soil moisture measurements by TDR. J. Soil Sci. 43:1-13.

Stein, J. and D.L. Kane. 1983. Monitoring the unfrozen water content of soil and snow using time domain reflectometry. Water Resources Res. 19(6):1573-1584

Topp, G.C., J.L. Davis, and A.P. Annan. 1980 Electromagnetic determination of soil water content: Measurements in coaxial transmission lines. Water Resources Res. 16(3):574-582.

Van Loon, W.K.P.,E. Perfect, P.H. Groenevelt, and B.D. Kay. 1990. A new method to measure bulk electrical conductivity in soils with time domain reflectometry. Can. J. Soil Sci. 70:403-410.

Wang, J.R. and T.J. Schmugge. 1980. An empirical model for the complex dielectrical permittivity of soils as a function of water content. IEEE Trans. Geoscience Remote Sensing GE-18:288 295. 